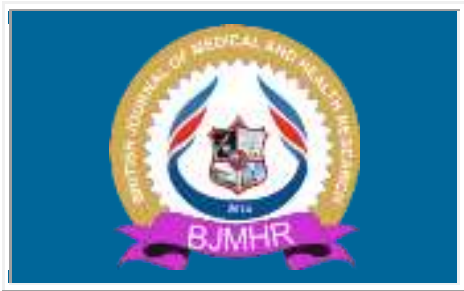

\title{
BJMHR
}

British Journal of Medical and Health Research

Journal home page: www.bjmhr.com

\section{Role of Physical Activity In Breast Cancer}

Anil Batta

Department of Medical Biochemistry, Govt. Medical College

\section{ABSTRACT}

Breast cancer is the most common cancer among women. The number of breast cancer survivors has increased due to screening and improved treatment methods, which makes it important to increase knowledge on their health and well-being. Physical activity has been reported to improve quality of life, decrease fatigue and reduce all-cause and breast cancer-specific mortality in breast cancer survivors. In physically active subjects, the risk reduction averaged $25-30 \%$. Breast cancer is one of the most commonly diagnosed types of cancer in women. Its pathogenesis involves genetic, hormonal, and environmental factors ${ }^{6}$. A large body of evidence indicates that physical activity has positive effects on every aspect of breast cancer evolution, including prevention, medical treatment, and aftercare clinical settings. Thus, different types of exercise can influence the prevention and progression of the disease through several common mechanisms, such as reduction of insulin resistance and improvement of immunity and cardiovascular function. Furthermore, acute and chronic symptoms of breast cancer, such as cachexia, muscle mass loss, fatigue, cardiotoxicity, weight gain, hormone alterations, bone loss, and psychological adverse effects, may all be favorably influenced by regular exercise. ${ }^{7}$ The potential role of exercise in reducing the intensity of the adverse effects that result from breast cancer and anticancer treatment is also discussed ${ }^{1}$. According to published data, 30$60 \mathrm{~min}$ of exercise per day at a moderate intensity is regarded as the optimal duration. Furthermore, physical activity plays an important role in the therapy of breast cancer, not only after finishing but already during treatment. The beneficial effects of physical activity may manifest themselves in circulating levels of insulin, insulin-growth factors (IGFs) I and II and their binding proteins (IGFBPs), or inflammatory biomarkers. The aim of this report was to review available randomized controlled trials (RCTs) on the effects of physical activity on biomarkers in breast cancer survivors. Five RCTs reported statistically significant effects of physical activity on insulin, IGF-I, IGF-II and IGFBP-3 in breast cancer survivors, but the results were not consistent. None of four RCTs found any evidence for a role of investigated interleukins. One trial reported some evidence that exercise may decrease C-reactive protein levels. In conclusion, available RCTs have produced some evidence that physical activity may result in beneficial changes in levels of insulin, IGFs, IGFBPs, and inflammatory biomarkers in breast cancer survivors. However, further larger RCTs on physical activity and biomarkers in breast cancer survivors are warranted.

Keywords: Physical activity, Intervention, Breast cancer survivors, Insulin, Insulin-growth factors, Inflammation 


\section{INTRODUCTION}

Women diagnosed with breast cancer are administered complex oncological treatment composed of surgical treatment combined with radiotherapy, chemotherapy and hormone therapy, depending on the stage and type of cancer ${ }^{11}$ The majorities of women who enter into cancer treatment give up physical activity or perform only light intensity activities. The evidence available today suggests that aerobic training might be safe and effective even during chemotherapy. Hornsby et al. ${ }^{6}$ proved the beneficial effects of physical exercise on a group of patients diagnosed with breast cancer who were in the course of chemotherapy (doxorubicin and cyclophosphamide) ${ }^{8}$. First of all, they found that it is safe and tolerated well by the patients to include high intensity aerobic training in traditional chemotherapy (based on anthracycline). Secondly, the application of solely anthracycline-based chemotherapy clearly impairs the function of the circulatory and respiratory systems. Thirdly, aerobic training eliminates the negative effects of chemotherapy on the body and improves the function of the circulatory and respiratory systems in the course of neoadjuvant therapy. Another issue is that some of the patient-reported outcomes (PROs), measured on FACT-B scale, are significantly improved ${ }^{11}$. Those outcomes include: quality of life, fatigue and depression measured in the course of cancer treatment and thereafter ${ }^{[6]}$. The authors of many pending searches of literature and meta-analyses conclude that aerobic training is a safe and efficient way improve the function of the circulatory and respiratory systems - it reduces fatigue and improves the quality of life of patients who are in the course of or have already completed a cancer treatment ${ }^{12,13 .}$ In a meta-analysis of Schmitz et al. ${ }^{[12],}$ the authors point to a series of research studies on the effects of physical activity on patients with breast cancer who are in the course of or have already completed treatment. They selected 22 randomized samples where the safety and efficiency of physical exercise in patients in the course of chemo- and radiotherapy were revealed. Furthermore, the results of 10 of those studies pointed to a significant improvement of the oxygen capacity in the patients, and 5 pointed to the statistically significant improvement of muscle strength ${ }^{6,9}$. In the following step, they analyzed the impact of physical activity on the dimensions of the body, its composition and the quality of life and level of fatigue in the patients. The authors found a decreased body weight and BMI values, improved body composition in 6 samples, while in 4 of those the quality of life of patients subject to cancer treatment was found to have improved, and in 7 the physical exercise was found to have reduced fatigue. Furthermore, the results of some of the research studies indicated that physical exercise may have an effect on physical functions, bone density, the range of motion in the shoulder joint, sleeping and the level of blood hemoglobin ${ }^{13}$. Exercise was found to be beneficial in patients who have already completed 
cancer treatment, as well. In all of the 32 research studies conducted on patients who have completed therapy, the oxygen capacity has improved. ${ }^{3}$ In addition, the results of some of the patients pointed to improved muscle strength and flexibility, improved dimensions, body composition and quality of life ${ }^{12}$. Physical activity plays an important role in case of women who are administered only local treatment. Stan et al ${ }^{[1,4]}$ found that women who had had a mastectomy and followed a special individualized exercise plan based on the Pilates system for 12 weeks had a significantly improved abduction movement in the shoulder joint. Sandel et al. reached similar conclusions ${ }^{2}$. They administered dance therapy to women after surgery. During the experiment, the women did a 12-week training programmed and afterwards were subject to assess Improvement in survival over the past 20 years has resulted in a substantial number of breast cancer survivors, many of whom will have a normal life expectancy. ${ }^{-3}$ Increasingly, cancer care is being

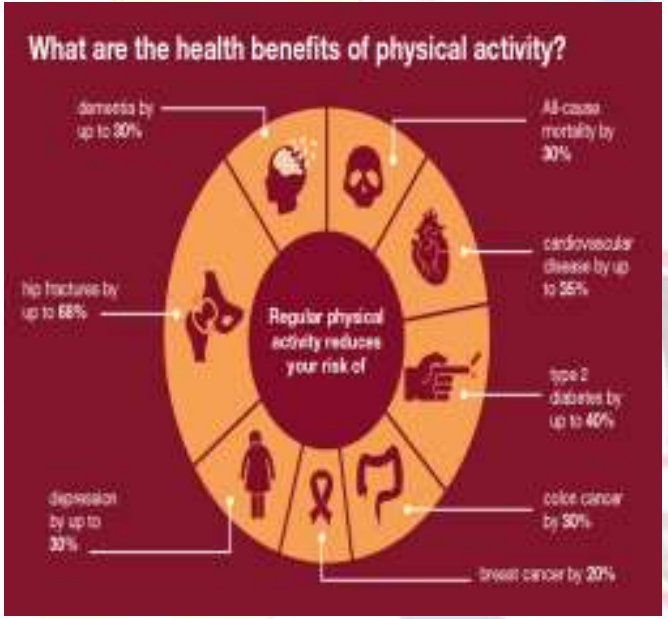

Regular Physical Activity Health Benefits in Children

$>$ Helps build healthy bones and muscles.

Helps reduce the risk of developing obesity and chronic diseases, such as diabetes and cardiovascular disease.

$>$ Reduces feelings of depression and anxiety and promotes psychological well-being.

-Can help improve students' academic performance, including: (1) academic achievement and grades, (2)academic behavior, such as time on task, and (3) factors that influence academic achievement, such as concentration and attentiveness in the classroom.

Arter

Exercise after chemo satety, aerobic fitness, muscular strength, body size and composftion, OOL, fatigue, arixiety

After Breast Cancer treatment

Safety: 15 RCTs advocated exercise Body size and composition. There after breast cancer however there. Was wrak evidence to support this bit was general consensus that $\quad$ out of the 16 studies reviewed haf of physiotherapist should be aware of them snowed statistical signticant exercise presenption particulany. evidence in at leas one or moore of the with arms and shouiders in breast lohowing variabies, BMi, fat mass, lean

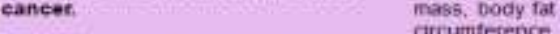

Aerobic fitness: 10 studies using Fatigue: 2 RCTs examined the efted of exeroise interventions using timed exerose on fatigue and 4 studies found waik test and maximal oxygen uptake an improvement while the remaining showed statistical significance in Museutar strength: All six resistance exercise trials showed statistical significant difference in both upper and lower body strength post treatiment studies tound no significart differe
but shomed no adverse effects. QeL: Numerous studies using breast cancer sulascale of functional assessment of cancer therapy nave supported the view that exerche improved gaL.

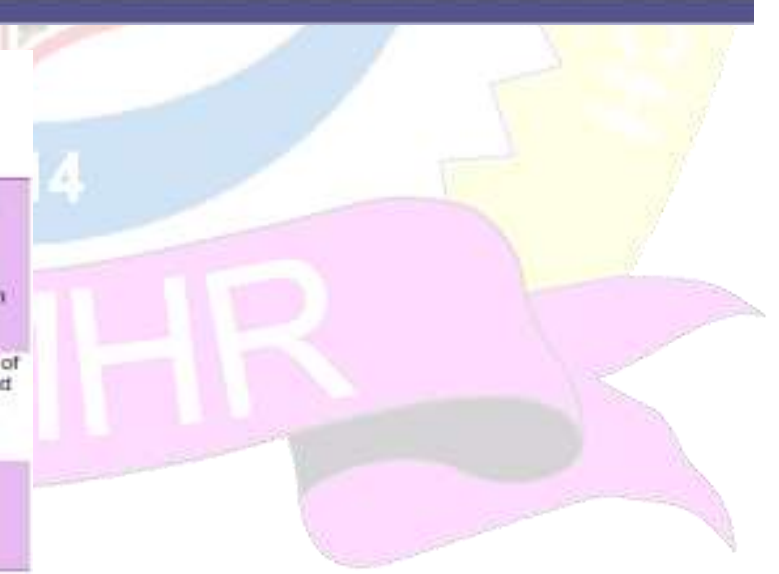


directed toward developing interventions to improve overall quality of life as well as
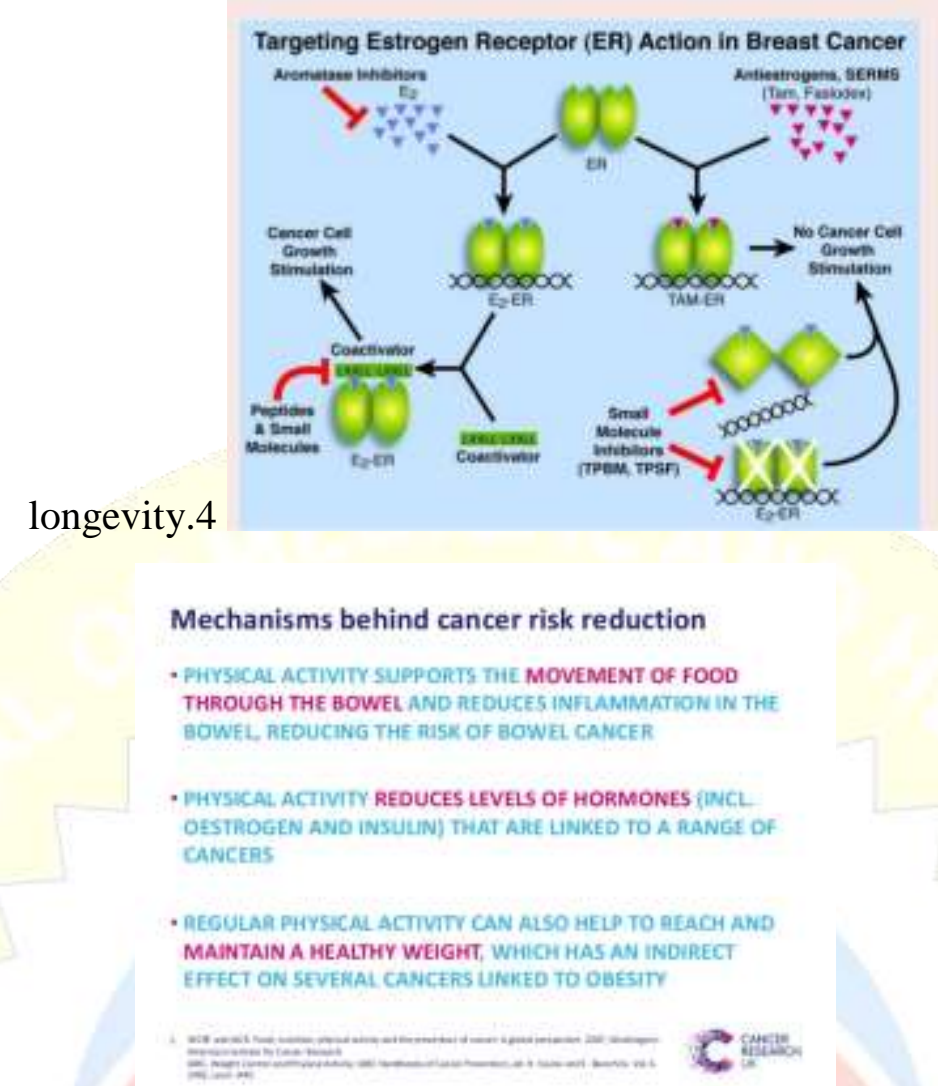

Physical exercise has consistently been identified as a central element of rehabilitation for many chronic diseases $\frac{5-8}{}$ and has been successful in improving quality of life and reducing all-cause mortality. ${ }^{9}$ Recent observational evidence suggests that moderate levels of physical activity may even reduce the risk of death from breast cancer, ${ }^{10}$ and therefore exercise may prove to be a valuable intervention to improve not only quality of life but overall survival.

The effectiveness of exercise interventions in cancer patients and survivors has been assessed in both qualitative systematic reviews and meta-analyses that included all types of cancers and all types of trial designs (i.e., nonrandomized, uncontrolled trials). ${ }^{11-13}$ It is well known, however, that cancer survivor groups are clinically heterogeneous in terms of their demographic profile (e.g., age, sex distribution), behavioral profile (e.g., smoking status, alcohol consumption, obesity), disease pathophysiology, treatment protocols, and symptoms and side effects. Consequently, the wisdom of summarizing the effects of exercise interventions across such disparate groups is questionable ${ }^{3}$. It is clear from previous reviews that the vast majority of exercise intervention research has involved breast cancer patients and survivors ${ }^{7}$. In addition, there are now newer studies, so there is sufficient research available to restrict a meta-analysis to this cancer survivor group ${ }^{2}$. It is also well known that the inclusion of nonrandomized or uncontrolled trials leads to an overestimation of the effect of an intervention. It is recommended that meta-analyses be restricted to randomized 
controlled trials (RCTs) whenever possible. ${ }^{14}$ Here, we present a systematic quantitative review of RCTs on the effects of exercise interventions on breast cancer patients and survivors $^{8 .}$

\section{DISCUSSION}

Considerable research interest has been given in the past 25 years to examining the role of physical activity in breast cancer prevention given the scarcity of modifiable risk factors for this major cause of cancer incidence and mortality in women ${ }^{2}$, 3. A review of the observational epidemiologic evidence and recent randomized exercise intervention trials on the association between physical activity and breast cancer risk is presented ${ }^{2}$. There also was evidence for a dose-response effect found in the majority of studies that examined this trend. The strongest associations were found for recreational and household activities and for activity that was of at least moderate intensity and sustained over a lifetime. Within population subgroups, a stronger effect was seen in women who are normal weight, in women without a family history of breast cancer, and in women who are parous ${ }^{13}$. Women of all races benefitted from physical activity; however, a particularly strong effect on breast cancer risk was observed in non-Caucasian women. ${ }^{3}$ Future research should focus on elucidating the exact type, dose, and timing of physical activity required to reduce breast cancer risk. Prospective observational epidemiologic studies of lifetime physical activity patterns and breast cancer risk would help in this regard, as well as randomized controlled exercise intervention trials employing hypothesized biomarkers of breast cancer risk as outcome measures ${ }^{4}$. Additional consideration to the role of sedentary behavior and lightintensity activity also is needed, as well as improved physical activity assessment methods. These additional data will be useful in improving public health recommendations regarding physical activity for breast cancer risk reduction ${ }^{5}$. At present, it is not yet possible to describe conclusively the interlocking biochemical pathways. Nevertheless, different possible influences shall be presented. First of all, acute and chronic workloads (in the sense of training) have an impact on various parts of the immune system. It is assumed that this is caused by the exercise-induced release of hormones, in particular catecholamines and cortisol $^{2}$. As a consequence, the number of cells and, moreover, the function of cellular and humoral defense mechanisms change. This is mainly understood with regard to natural killer cells or cytotoxic $\mathrm{T}$ cells, but also to transmitters of the immune system, the so-called cytokines (e.g. C-reactive protein (CRP) ${ }^{[7-9]}$. Among other things, catecholamines lead to a dose-dependent augmentation of natural killer (NK) cells by approximately 150-300\% and of cytotoxic $\mathrm{T}$ cells by $50-100 \%{ }^{[10]}$. However, within the first $2 \mathrm{~h}$ after workload, a strong decrease is observed. Basically, this effect occurs when cortisol is released, and it is an 
explanation why high load intensities provoke more prominent reactions ${ }^{8}$. The low levels of monocytes and macrophages seen after intensive workloads sometimes even remain depleted for several days (s. 'open window'). The function of the NK cells may enhance by around $40-100 \%$ and decrease - according to the intensity-by around $25-35 \%$ below baseline ${ }^{[11]}$. The phagocytic and cytotoxic functions of the macrophages seem to be enhanced especially after moderate physical activities ${ }^{5}$. These findings do not yet provide sufficient evidence, but support the hypothesis that moderate physical activities in particular have a positive impact on the immune system ${ }^{[12,13] .}$ In addition, physical activity has great effects on sex hormones. Active women show lower concentrations of sex hormones and, hence, a decreased exposure to free estrogen ${ }^{[13,14] .}$ Estrogens promote the risk of mammary cancer development, which is attributed to the stimulation of mitoses and the regulation of cell proliferation [12]. Progesterone and androgens may also favor the development of tumors. Before menopause, the ovaries are the primary source of estrogen synthesis, whereas after menopause aromatized ovarian and adrenal androgens mainly contribute to the estrogen production. By aromatase, androgen precursor substances (testosterone, androstendione) are converted to estrogens [2]. This conversion occurs at extragonadal sites, predominantly in visceral adipose tissue ${ }^{2}$; therefore, the body composition, and especially the percentage of body fat, plays an important role in the prevention of developing breast cancer after menopause. Meanwhile, in the therapy of breast cancer patients, the administration of aromatase inhibitors is well established. They inhibit the conversion of androgens to estrogens. In the context of body composition and visceral adipose tissue, the increased concentration of insulin is discussed, which influences the bioavailability of estrogens and androgens by down-regulating sex hormone-binding globulin (SHBG) and up-regulating ovarian hormone production. However, it should be considered that the growth hormones insulin and insulin-like growth factor-1 (IGF-1) they have an impact on carcinogenesis. Goodwin et al. ${ }^{[2]}$ reported a relationship between higher levels of fasting insulin and an adverse prognostic factor for breast cancer in 512 women with diabetes mellitus. The tumorigenic effects of insulin can be directly relayed by insulin receptors to (pre-) neoplastic cells or they are a secondary consequence of hyperinsulinemia which leads to changes of the endogenous hormone metabolism. Insulin, for example, enhances the synthesis and biological actions of IGF-1 and thus is found to be an important factor in the pathogenesis of tumor diseases ${ }^{[2,3] .}$ It is a potent mitogen that plays a decisive role in regulating apoptosis, proliferation, and differentiation of cells. According to a large number of epidemiological studies, this effect is associated with a higher incidence of mammary cancer risk ${ }^{[2,4] .}$ The effects of IGF-1 are mainly mediated by the IGF-1 receptor. The latter is very similar to the insulin receptor (60\% homology). For this reason, both insulin 
and IGF-1 are able to activate both receptors. In addition, the effect of IGF-1 is regulated by IGF-binding proteins (IGFBPs). It has been scientifically proven that physical activity leads to improved insulin sensitivity, to a reduced level of insulin, and to an increased concentration of IGFBP-3 ${ }^{[6,7]}$

\section{SUMMARY}

More and more recent studies have identified the association between the adipocytokines leptin and adiponectin and the development of breast cancer. They represent 2 of around 100 factors that are secreted from visceral adipose tissue ${ }^{[2,8]}$. Leptin was detected in breast cancer tissue and in breast cancer cell lines and correlated positively with the body fat mass ${ }^{[2,3]}$. It is assumed that leptin stimulates mammary tumor cell growth. The biological effect of leptin on the development and distribution of breast cancer can be attributed to the leptin-induced proliferation of mammary tumor cell lines, the increase of proteolytic enzymes that are necessary in the process of dissemination, and the stimulating effect on angiogenesis. The impact of aromatase inhibitors on leptin is not yet well understood. Adiponectin, however, shows opposite effects; it appears to enhance the insulin sensitivity ${ }^{[3,5]}$ and to have an anti

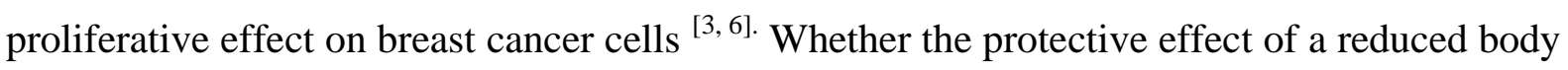
fat mass could be the decisive factor has not yet been sufficiently studied. However, it was reported that the adiponectin concentration was negatively associated with the body fat mass and increased when the body fat mass declined ${ }^{[3,7]}$.

Regarding cellular pathways, inflammatory processes are thought to play a pivotal role. Physical activity influences both systemically and locally the balance between pro-and antiinflammatory cytokines ${ }^{[13]}$. Further effects that probably depend on the duration and intensity of the respective exercise workload take place in signaling pathways, e.g. the effects of 5'AMP-activated protein kinase (AMPK), phosphoinositide 3-kinase (PI3K), MAPK, and NFkappaB [3,9]. These signaling pathways are extremely important when studying the development of mammary cancer as they are involved in different ways in the regulation of transcription, in the development of insulin resistance, apoptosis, infection, etc. However, it is not yet understood whether the influencing processes already take place on the DNA level ${ }^{[4]}$.

\section{CONCLUSION}

It is generally believed that physical activity is closely associated with the reduction of risk of development of breast cancer as well as colorectal cancer, prostate cancer, endometrial cancer and lung cancer. Physical exercise lowers the risk of occurrence of carcinogens ${ }^{[3,2] .}$ There is substantial scientific evidence for the lowered risk of development of the disease caused by moderately intense physical activity ${ }^{[9-13]}$. Regular physical activity among current patients or patients who have already completed treatment may significantly decrease the risk of death 
[3]. Researchers name a lot of mechanisms associated with the effects of physical activity on the risk of development of breast cancer and the pace of recovery, but further research is still required ${ }^{[3,4]}$.

\section{REFERENCES}

1. DeSantis C, Siegel R, Bandi P, Jemal A. Breast cancer statistics, 2011. CA: a cancer journal for clinicians. 2011; 61:409-418.

2. Duijts SF, Faber MM, Oldenburg HS, van Beurden M, Aaronson NK. Effectiveness of behavioral techniques and physical exercise on psychosocial functioning and health-related quality of life in breast cancer patients and survivors--a metaanalysis. Psychooncology. 2011;20:115-126.

3. Schmitz KH, Speck RM. Risks and benefits of physical activity among breast cancer survivors who have completed treatment. Women's health. 2010;6:221-238.

4. Schmitz KH, Courneya KS, Matthews C, Demark-Wahnefried W, Galvao DA, Pinto BM, et al. American College of Sports Medicine roundtable on exercise guidelines for cancer survivors. Medicine and science in sports and exercise. 2010; 42:1409-1426.

5. Irwin ML, McTiernan A, Manson JE, Thomson CA, Sternfeld B, Stefanick ML, et al. Physical activity and survival in postmenopausal women with breast cancer: results from the women's health initiative. Cancer prevention research. 2011; 4:522-529.

6. Holmes MD, Chen WY, Feskanich D, Kroenke CH, Colditz GA. Physical activity and survival after breast cancer diagnosis. Jama. 2005; 293:2479-2486.

7. Holick CN, Newcomb PA, Trentham-Dietz A, Titus-Ernstoff L, Bersch AJ, Stampfer MJ, et al. Physical activity and survival after diagnosis of invasive breast cancer. Cancer epidemiology, biomarkers \& prevention : a publication of the American Association for Cancer Research. cosponsored by the American Society of Preventive Oncology. 2008; 17:379-386.

8. Irwin ML, Smith AW, McTiernan A, Ballard-Barbash R, Cronin K, and Gilliland FD, et al. Influence of pre- and postdiagnosis physical activity on mortality in breast cancer survivors: the health, eating, activity, and lifestyle study. Journal of clinical oncology: official journal of the American Society of Clinical Oncology. 2008; 26:3958-3964.

9. Sternfeld B, Weltzien E, Quesenberry CP, Jr., Castillo AL, Kwan M, Slattery ML, et al. Physical activity and risk of recurrence and mortality in breast cancer survivors: findings from the LACE study. Cancer epidemiology, biomarkers \& prevention: a publication of the American Association for Cancer Research, cosponsored by the American Society of Preventive Oncology. 2009; 18:87-95 
10. Hess KR, Pusztai L, Buzdar AU, Hortobagyi GN. Estrogen receptors and distinct patterns of breast cancer relapse. Breast cancer research and treatment. 2003; 78:105118.

11. Voduc KD, Cheang MC, Tyldesley S, Gelmon K, Nielsen TO, Kennecke H. Breast cancer subtypes and the risk of local and regional relapse. Journal of clinical oncology : official journal of the American Society of Clinical Oncology. 2010; 28:1684-1691.

12. Wang Y, Yin Q, Yu Q, Zhang J, Liu Z, Wang S, et al. A retrospective study of breast cancer subtypes: the risk of relapse and the relations with treatments. Breast cancer research and treatment. 2011; 130:489-498.

13. Iturbe J, Zwenger A, Leone JP, Verdera PP, Vallejo C, Romero A, et al. Treatment of early breast cancer, a long-term follow-up study: the GOCS experience. Breast J. 2011; 17:630-637.

14. Hess KR, Esteva FJ. Effect of HER2 status on distant recurrence in early stage breast cancer. Breast cancer research and treatment. 2013; 137:449-455.

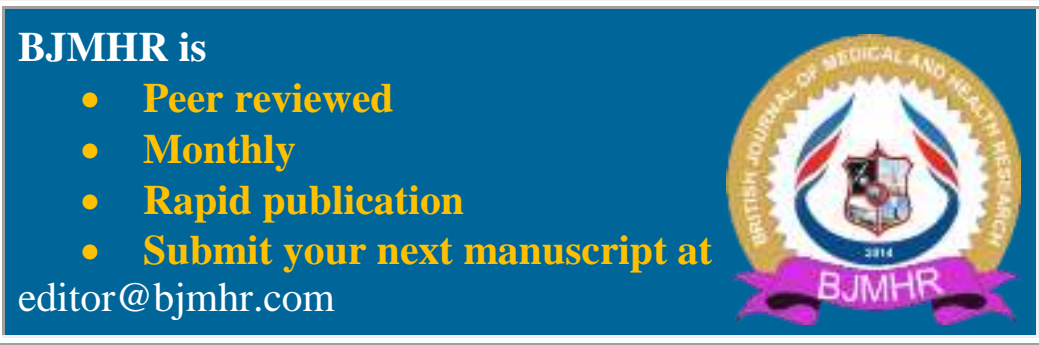

